\title{
Nutritional jejunostomy in esophagectomy for cancer, a national register-based cohort study of associations with postoperative outcomes and survival
}

\author{
Anders Holmén ${ }^{1,2}$ (D) Masaru Hayami ${ }^{1,3} \cdot$ Eva Szabo $^{4,5} \cdot$ loannis Rouvelas ${ }^{1,3} \cdot$ Thorhallur Agustsson $^{2,6}$. \\ Fredrik Klevebro ${ }^{1,3}$
}

Received: 28 July 2020 / Accepted: 17 November 2020 / Published online: 24 November 2020

(C) The Author(s) 2020

\begin{abstract}
Purpose Insertion of a nutritional jejunostomy in conjunction with esophagectomy is performed with the intention to decrease the risk for postoperative malnutrition and improve recovery without adding significant catheter-related complications. However, previous research has shown no clear benefit and there is currently no consensus of practice.

Methods All patients treated with esophagectomy due to cancer during the period 2006-2017 reported in the Swedish National Register for Esophageal and Gastric Cancer were included in this register-based cohort study from a national database. Patients were stratified into two groups: esophagectomy alone and esophagectomy with jejunostomy.

Results A total of 847 patients (45.27\%) had no jejunostomy inserted while 1024 patients (54.73\%) were treated with jejunostomy. The groups were comparable, but some differences were seen in histological tumor type and tumor stage between the groups. No significant differences in length of hospital stay, postoperative surgical complications, Clavien-Dindo score, or 90-day mortality rate were seen. There was no evidence of increased risk for significant jejunostomy-related complications. Patients in the jejunostomy group with anastomotic leaks had a statistically significant lower risk for severe morbidity defined as Clavien-Dindo score $\geq \mathrm{IIIb}$ (adjusted odds ratio 0.19, 95\% CI: 0.04-0.94, $P=0.041$ ) compared to patients with anastomotic leaks and no jejunostomy.

Conclusion A nutritional jejunostomy is a safe method for early postoperative enteral nutrition which might decrease the risk for severe outcomes in patients with anastomotic leaks. Nutritional jejunostomy should be considered for patients undergoing curative intended surgery for esophageal and gastro-esophageal junction cancer.
\end{abstract}

Keywords Esophageal cancer $\cdot$ Esophagectomy $\cdot$ Postoperative complications $\cdot$ Anastomotic leak $\cdot$ Feeding jejunostomy

Anders Holmén

anders.holmen@ki.se

1 Department of Clinical Science, Intervention and Technology (CLINTEC), Karolinska Institutet, Stockholm, Sweden

2 Department of Surgery, Södersjukhuset, Stockholm, Sweden

3 Department of Upper Abdominal Cancer, Karolinska University Hospital, Stockholm, Sweden

4 Department of Surgery, Örebro University Hospital, Örebro, Sweden

5 School of Medical Sciences, Örebro University, Örebro, Sweden

6 Department of Clinical Science and Education, Karolinska Institutet | Södersjukhuset, Stockholm, Sweden

\section{Introduction}

Postoperative complications after esophagectomy are common [1] and associated with increased mortality [2-4] and long-term decreased health-related quality of life (HRQOL) [5, 6].

Esophagogastric anastomotic leak is the major complication after esophagectomy, with a reported incidence of 4-35\% $[1,7,8]$. Patients who suffer an anastomotic leak have an almost tenfold increase in the 30-day mortality rate from 2$3 \%$ to $17-35 \%$ [2]. Anastomotic leaks are associated with a nutritional deficit, which can make recovery difficult.

Nutrition is fundamental for the successful treatment of esophageal cancer where malnutrition, weight loss, and cancer cachexia are particularly prevalent [9]. Early postoperative enteral nutrition has proven to be clearly beneficial and is a key component of the ERAS protocol [10]. Enteral nutrition is 
associated with improved levels of gut oxygenation, lower costs, and reduced postoperative length of stay compared with total parenteral nutrition [11-13].

Insertion of a nutritional jejunostomy is made with the intent to secure a nutritional route, should postoperative oral feeding be contraindicated or insufficient. The jejunostomy can be used to give full enteral nutrition after surgery or for nutritional support in combination with early oral feeding depending on the applied clinical pathway. There is a risk for catheter-related complications, most of which are minor, such as local skin contamination, dislocation, catheter site infection, and occlusion [14]. Severe complications such as small bowel necrosis and intestinal torsion are rare but potentially life-threatening [15-17].

The aim of this study was to determine if the insertion of a nutritional jejunostomy in conjunction with esophagectomy for cancer was associated with decreased postoperative morbidity. The secondary outcome was to evaluate if the opportunity to give enteral nutrition with the use of a jejunostomy was associated with improved outcomes for patients with the postoperative anastomotic leak.

\section{Methods}

\section{Study design}

A nationwide, retrospective, population-based cohort study from a prospectively collected national database including all patients undergoing esophageal cancer surgery in Sweden between 2006 and 2017 was performed. Data was collected from the Swedish National Register for Esophageal and Gastric Cancer, in which all patients with esophageal or gastroesophageal cancer in Sweden are included. The register has a national coverage of $95.5 \%$ and an overall accuracy of $91 \%$ [18]. The clinical data include patient and tumor characteristics, treatment details regarding oncological and surgical management (including the insertion of a jejunostomy or not), and study outcomes.

\section{Exposure}

Study exposure was the insertion of nutritional jejunostomy in conjunction with esophagectomy for cancer of the esophagus or the gastro-esophageal junction.

\section{Outcomes}

All clinical data were collected from the register. Enrolled patients were cross-matched with the National Cause of Death Register via the individual unique personal identification number assigned to all Swedish residents [19]. Outcomes included overall postoperative complications stratified by surgical or non-surgical complications, with surgical complications defined as follows: Postoperative leakage was confirmed with CT scan, with an oral water-soluble contrast medium, or verified with endoscopy. Conduit necrosis was defined as confirmed ischemia of the conduit with perforation or ulcer. Bleeding was defined as blood loss of more than $2 \mathrm{~L}$ or need of surgical re-intervention. Chylothorax was defined as a leak that required drainage for more than 7 days or a need for surgical re-intervention. Recurrent nerve paralysis was confirmed by an otorhinolaryngologist. Abdominal or thoracic abscesses were reported when the size of the abscess exceeded $3 \times 3 \mathrm{~cm}$ and was verified radiologically or surgically.

Included among the non-surgical complications were cardiac arrhythmias requiring medical treatment, myocardial infarction, and cerebral embolism. Pulmonary embolism was defined as radiographically confirmed embolus requiring treatment. The definition of respiratory failure was when patients required invasive or non-invasive ventilation. Pneumonia was defined as x-ray-confirmed infiltration combined with fever, cough, and/or dyspnea and infections nonrelated to the operation field. Septicemia was defined as a body temperature above $38.3{ }^{\circ} \mathrm{C}\left(101{ }^{\circ} \mathrm{F}\right)$ or below $36{ }^{\circ} \mathrm{C}$ $\left(96.8^{\circ} \mathrm{F}\right)$ with a positive blood culture. Length of hospital stay in days and overall all-cause mortality were calculated based on data from the National Cause of Death Register.

\section{Statistical methods}

Multivariable logistic regression modeling, chi-square test, and Fisher's exact test were used for binomial outcomes. The multivariable logistic regression model and the Cox proportional hazard model were pre-specified and included tumor histology, clinical tumor stage, tumor location, and ASA score. Complete case analysis was performed in the multivariable-adjusted model. The categorizations of the variables are displayed in Table 1. The Cox proportional hazard model was used for the survival analyses. The proportional hazard assumptions were tested in all models using the Grambsch and Therneau test based on Schoenfeld residuals, which did not show any violations. For each outcome, we report the odds ratio (OR) and 95\% confidence interval (CI). The significance level was set at 0.05 . Analyses were performed using STATA ${ }^{\circledR}$ version 13 software (StataCorp LP, College Station, Texas, USA).

\section{Results}

\section{Patient demographics and baseline characteristics}

Out of the 1871 patients who underwent surgery for esophageal or gastro-esophageal junction cancer, 847 (45.3\%) were treated with no jejunostomy and $1024(54.7 \%)$ with nutritional jejunostomy. The groups were similar with regard to age, 
Table 1 Baseline characteristics of patients undergoing esophagectomy for esophageal or gastro-esophageal junction cancer, stratified by nutritional jejunostomy

\begin{tabular}{|c|c|c|c|}
\hline$n(\%)$ & No jejunostomy & Jejunostomy & $P$ value \\
\hline Total & $847(45.3)$ & $1024(54.7)$ & \\
\hline Age, median (range) & $66(20-93)$ & $66(29-88)$ & 0.999 \\
\hline Gender & & & 0.763 \\
\hline Male & $671(79.2)$ & $817(79.8)$ & \\
\hline Female & $176(20.8)$ & $207(20.2)$ & \\
\hline Mean body weight in kg (range) & $80.8(47-141)$ & $81.3(51-137)$ & 0.999 \\
\hline Performance status & & & 0.104 \\
\hline 0 & $452(57.1)$ & $598(61.9)$ & \\
\hline 1 & $291(36.8)$ & $322(33.3)$ & \\
\hline 2 & $48(6.1)$ & $46(4.8)$ & \\
\hline Unknown & 56 & 58 & \\
\hline ASA score & & & 0.458 \\
\hline I & $292(35.9)$ & $347(35.6)$ & \\
\hline II & $397(48.8)$ & $475(48.7)$ & \\
\hline III & $116(14.3)$ & $150(15.4)$ & \\
\hline IV & $8(1.0)$ & $4(0.4)$ & \\
\hline Unknown & 34 & 48 & \\
\hline Baseline dysphagia score & & & 0.765 \\
\hline No dysphagia & $76(32.8)$ & $64(30.9)$ & \\
\hline Dysphagia to solid food & $108(46.6)$ & $103(49.8)$ & \\
\hline Dysphagia to semi-solid food & $33(14.2)$ & $30(14.5)$ & \\
\hline Dysphagia to liquids & $14(6.0)$ & $8(3.9)$ & \\
\hline Total dysphagia & $1(0.4)$ & $2(1.0)$ & \\
\hline Unknown & 615 & 817 & \\
\hline
\end{tabular}

gender, body weight, performance status, ASA score, and dysphagia score (Table 1).

\section{Tumor characteristics and treatment details}

Jejunostomies were less frequently inserted in cT1 tumors (12.5\% vs. $7.0 \%$ ) and slightly more often in T2 and T3 tumors $(25.4 \%$ vs. $29.1 \%, 46.5 \%$ vs. $48.1 \%, P=0.002)$. No significant differences were found concerning clinical $\mathrm{N}$-stage, tumor location, preoperative treatment, or surgical approach. Jejunostomy was more often used with a transthoracic and transhiatal approach compared to gastrectomy $(P=0.001$, Table 2$)$. Histological tumor type was different between the groups $(P=0.005)$, with a higher tumor burden in the jejunostomy group. There was no significant difference in neoadjuvant treatment. Jejunostomies were inserted with open technique in 852 $(83.2 \%)$ patients and laparoscopic technique in 172 patients $(16.8 \%$, Table 3$)$. The register had some missing data concerning clinical T-stage (219 patients, $11.7 \%$ ), $\mathrm{N}$-stage (92 patients, $4.9 \%$ ), tumor location (220 patients, $11.8 \%$ ), surgical technique (98 patients, 5.2\%), and histological tumor type (24 patients, $1.3 \%$ ) (Table 2 ).

\section{Short-term clinical outcomes and survival}

Postoperative complications were reported in 330 (39.0\%) patients with no jejunostomy and in 454 (44.4\%) patients with jejunostomy $(P=0.019)$. No significant differences in severity of complications according to the Clavien-Dindo scoring system were observed. Missing data concerning Clavien-Dindo score was reported in 211/784 (26.9\%) of the patients with complication. The median length of hospital stay was similar. No significant differences were seen concerning the incidence of anastomotic leak, gastric conduit necrosis, re-operations, or occurrences of thoracic abscesses. Recurrent laryngeal nerve paralysis was more frequently reported in patients with jejunostomy $(2.5 \%$ vs $4.3 \%, P=0.033)$ as were non-surgical complications $(22.2 \%$ vs $29.8 \%, P<0.001)$ such as pneumonia (7.2\% and $11.8 \%, P=0.001$ ). The postoperative 90-day mortality was $6.9 \%$ in patients without jejunostomy and $5.1 \%$ in the jejunostomy group $(P=0.105$, Table 3$)$.

No significant differences were observed comparing open to laparoscopic jejunostomy concerning postoperative complications, surgical complications, re-operations, or ClavienDindo score $\geq$ IIIb (Table 3 ).

Multivariable adjusted analyses showed a statistically significant increase in risk for postoperative non-surgical 
Table 2 Tumor characteristics and treatment details of patients undergoing esophagectomy for esophageal or gastro-esophageal junction cancer, stratified by nutritional jejunostomy

\begin{tabular}{|c|c|c|c|}
\hline$n(\%)$ & No jejunostomy & Jejunostomy & $P$ value \\
\hline Clinical T-stage & & & 0.002 \\
\hline $\mathrm{T} 1$ & $106(12.5)$ & $72(7.0)$ & \\
\hline $\mathrm{T} 2$ & $215(25.4)$ & $298(29.1)$ & \\
\hline $\mathrm{T} 3$ & $394(46.5)$ & $493(48.1)$ & \\
\hline $\mathrm{T} 4$ & $35(4.1)$ & $39(3.8)$ & \\
\hline $\mathrm{TX}$ & $97(11.5)$ & $122(11.9)$ & \\
\hline Clinical N-stage & & & 0.653 \\
\hline N-negative & $452(53.4)$ & $568(55.5)$ & \\
\hline N-positive & $353(41.7)$ & $406(39.7)$ & \\
\hline NX & $42(5.0)$ & $50(4.9)$ & \\
\hline Tumor location & & & 0.154 \\
\hline Proximal & $21(2.5)$ & $24(2.4)$ & \\
\hline Middle & $69(8.2)$ & $115(11.4)$ & \\
\hline Distal & $467(55.6)$ & $575(56.9)$ & \\
\hline GE junction & $181(21.6)$ & $199(19.7)$ & \\
\hline Unknown & 109 & 111 & \\
\hline Preoperative treatment & & & 0.646 \\
\hline Surgery alone & $342(40.4)$ & $443(43.3)$ & \\
\hline Neoadjuvant chemotherapy & $146(17.2)$ & $175(17.1)$ & \\
\hline Neoadjuvant chemoradiotherapy & $345(40.7)$ & $385(37.6)$ & \\
\hline Definitive chemoradiotherapy & $14(1.7)$ & $21(2.1)$ & \\
\hline Surgical technique & & & 0.001 \\
\hline Transthoracic esophagectomy & $661(85.0)$ & $886(89.1)$ & \\
\hline Transhiatal esophagectomy & $30(3.9)$ & $46(4.6)$ & \\
\hline Gastrectomy & $87(11.2)$ & $63(6.3)$ & \\
\hline Unspecified & 69 & 29 & \\
\hline Surgical approach & & & 0.981 \\
\hline Open esophagectomy & $696(82.2)$ & $841(82.1)$ & \\
\hline Minimally invasive esophagectomy & $151(17.8)$ & $183(17.9)$ & \\
\hline Anastomosis level & & & 0.582 \\
\hline Thoracic & $749(88.4)$ & $897(87.6)$ & \\
\hline Cervical & 98 (11.6) & $127(12.4)$ & \\
\hline Histological tumor type & & & 0.005 \\
\hline Adenocarcinoma & $620(73.7)$ & $753(74.9)$ & \\
\hline Squamous cell carcinoma & $150(17.8)$ & $204(20.3)$ & \\
\hline Other & $71(8.4)$ & $49(4.9)$ & \\
\hline Unknown & 6 & 18 & \\
\hline
\end{tabular}

complications, pneumonia, septicemia, and recurrent nerve paralysis in the jejunostomy group (Table 4). There was no significant difference in long-term survival between the groups (Fig. 1).

\section{Clavien-Dindo score $\geq$ IIIb and 90-day mortality in patients with complications}

Any postoperative complication within 90 days was evaluated with adjusted logistic regression analysis to assess the impact on risk for Clavien-Dindo score $\geq \mathrm{IIIb}$, as well as 90-day postoperative mortality in patients with complications comparing the no jejunostomy group to the jejunostomy group. In patients with postoperative anastomotic leakage, the odds ratio for ClavienDindo score $\geq$ IIIb was 0.19 (95\% CI: 0.04-0.94) compared to patients without jejunostomy. There were no significant differences in the odds ratios for Clavien-Dindo score $\geq \mathrm{IIIb}$ concerning other postoperative complications or 90-day mortality. In patients with anastomotic leakage, the odds ratio for 90day mortality in the jejunostomy group was 0.53 (95\% CI: 0.24 
Table 3 Postoperative complications after esophagectomy for cancer, stratified by nutritional jejunostomy

\begin{tabular}{|c|c|c|c|}
\hline$n(\%)$ & No jejunostomy & Jejunostomy & $P$ value \\
\hline Postoperative complication & $330(39.0)$ & $454(44.4)$ & 0.019 \\
\hline Surgical complication & $223(26.3)$ & $282(27.5)$ & 0.557 \\
\hline Anastomotic leak & $78(10.5)$ & $115(12.6)$ & 0.195 \\
\hline Gastric conduit necrosis & $16(1.9)$ & $31(3.0)$ & 0.117 \\
\hline Postoperative bleeding & $17(2.0)$ & $15(1.5)$ & 0.368 \\
\hline Chylothorax & $26(3.1)$ & $26(2.5)$ & 0.487 \\
\hline Thoracic abscess & $21(2.5)$ & $39(3.8)$ & 0.104 \\
\hline Abdominal abscess & $11(1.3)$ & $13(1.3)$ & 0.955 \\
\hline Re-operation for any cause & $27(3.2)$ & $30(2.9)$ & 0.746 \\
\hline Recurrent laryngeal nerve paralysis & $21(2.5)$ & $44(4.3)$ & 0.033 \\
\hline Non-surgical complication & $188(22.2)$ & $305(29.8)$ & $<0.001$ \\
\hline Cardiovascular complication & $39(4.6)$ & $66(6.5)$ & 0.085 \\
\hline Pulmonary embolism & $16(1.9)$ & $31(3.0)$ & 0.117 \\
\hline Pneumonia & $61(7.2)$ & $121(11.8)$ & 0.001 \\
\hline Septicemia & $38(4.5)$ & $66(6.5)$ & 0.066 \\
\hline Clavien-Dindo score & & & 0.163 \\
\hline I & $46(18.5)$ & $59(18.2)$ & \\
\hline II & $74(29.7)$ & $110(34.0)$ & \\
\hline IIIa & $46(18.5)$ & $35(10.8)$ & \\
\hline IIIb & $47(18.9)$ & $56(17.3)$ & \\
\hline IVa & $23(9.2)$ & $40(12.4)$ & \\
\hline $\mathrm{IVb}$ & $6(2.4)$ & $10(3.1)$ & \\
\hline $\mathrm{V}$ & $7(2.8)$ & $14(4.3)$ & \\
\hline Unknown & 81 & 130 & \\
\hline Clavien-Dindo score $\geq$ IIIb & $83(33.3)$ & $120(37.0)$ & 0.358 \\
\hline Median length of hospital stay in days (IQR) & $15(10-23)$ & $16(12-24)$ & 0.032 \\
\hline 30-day mortality & $14(1.7)$ & $22(2.2)$ & 0.437 \\
\hline \multirow[t]{2}{*}{ 90-day mortality } & $58(6.9)$ & $52(5.1)$ & 0.105 \\
\hline & Open jejunostomy & Laparoscopic jejunostomy & \\
\hline Total & $852(83.2)$ & $172(16.8)$ & \\
\hline Postoperative complication & $378(44.4)$ & $76(44.2)$ & 0.965 \\
\hline Surgical complication & $228(26.8)$ & $282(27.5)$ & 0.557 \\
\hline Re-operation for any cause & $26(3.1)$ & $4(2.3)$ & 0.607 \\
\hline Abdominal abscess & $11(1.3)$ & $2(1.2)$ & 0.891 \\
\hline Clavien-Dindo score $\geq \mathrm{IIIb}$ & $79(38.2)$ & $41(35.0)$ & 0.576 \\
\hline
\end{tabular}

1.19) compared to patients with no jejunostomy. For patients with postoperative chylothorax, the corresponding odds ratio was 0.21 (95\% CI: 0.03-1.33, Table 5).

\section{Discussion}

The results of this population-based cohort study demonstrate that the insertion of a feeding jejunostomy in conjunction with esophagectomy was not associated with an overall increased risk for postoperative surgical complications. However, nonsurgical complications, such as pneumonia and septicemia, were more common in the jejunostomy group which might be explained by confounding factors such as surgical technique and increased use of jejunostomy for patients with more locally advanced tumors. The study shows that jejunostomy in patients with the anastomotic leak was associated with a significantly lower risk for Clavien-Dindo score $\geq \mathrm{IIIb}$, which suggests that jejunostomy in conjunction with esophagectomy might increase the chance to recover from an anastomotic leak without re-operation and intensive care. A jejunostomy provides a secure route for enteral nutrition in the event of an anastomotic leak which might explain the observed improved outcome in the jejunostomy group.

The observed higher incidence of pneumonia in the jejunostomy group is, to our knowledge, not previously 
Table 4 Multivariable adjusted logistic regression of postoperative complications comparing patients with jejunostomy vs. no jejunostomy in patients after esophagectomy for cancer

\begin{tabular}{|c|c|c|c|}
\hline & \multicolumn{3}{|c|}{ Odds ratio $(95 \% \text { confidence interval })^{\dagger}$} \\
\hline Any complication & 1.0 (reference) & $1.28(1.06-1.55)$ & 0.011 \\
\hline Surgical complication & 1.0 (reference) & $1.07(0.87-1.33)$ & 0.506 \\
\hline Anastomotic leak & 1.0 (reference) & $1.21(0.89-1.65)$ & 0.226 \\
\hline Chylothorax & 1.0 (reference) & $0.83(0.48-1.44)$ & 0.507 \\
\hline Recurrent laryngeal nerve paralysis & 1.0 (reference) & $1.94(1.11-3.38)$ & 0.020 \\
\hline Non-surgical complication & 1.0 (reference) & $1.53(1.23-1.90)$ & $<0.001$ \\
\hline Cardiovascular complication & 1.0 (reference) & $1.40(0.92-2.14)$ & 0.117 \\
\hline Pulmonary embolism & 1.0 (reference) & $1.72(0.92-3.21)$ & 0.089 \\
\hline Pneumonia & 1.0 (reference) & $1.79(1.29-2.48)$ & 0.001 \\
\hline Septicemia & 1.0 (reference) & $1.54(1.01-2.34)$ & 0.043 \\
\hline Clavien-Dindo $\geq \mathrm{IIIb}$ & 1.0 (reference) & $1.16(0.81-1.65)$ & 0.423 \\
\hline
\end{tabular}

$\dagger$ Adjusted for histological tumor type, clinical tumor stage, and American Society of Anesthesiologists Score demonstrated and is contradictory to results shown elsewhere $[20,21]$. Although it might reflect the association between jejunostomy and small bowel obstruction, as has been suggested by Koterazawa et al. [22], this was not seen in our study. Among surgical complications, the only statistically significant finding was an increased frequency of recurrent laryngeal nerve paralysis in the jejunostomy group. While this is unlikely to be related to the insertion of a jejunostomy, it is worth to notice. The increased incidence of pneumonia observed in the jejunostomy group might be explained by the higher incidence of recurrent laryngeal nerve paralysis in the jejunostomy group in terms of higher risk for aspiration. The results show a selection bias towards jejunostomy in more frail patients with a higher incidence of squamous cell carcinoma and a more advanced tumor stage. These patients require a more extensive lymph node dissection in the upper mediastinum which might explain the higher incidence of recurrent nerve palsy. The increased risk for non-surgical complications may also be explained by the increased use of jejunostomies in patients with more advanced tumor stages and in patients with the preoperative nutritional deficit, something that should be further assessed in future studies. Factors concerning baseline characteristics and type of surgery were included in the multivariable-adjusted model but there is a risk for residual confounding.

Data concerning preoperative nutritional status such as BMI and weight loss is not included in the register. Highquality data concerning weight loss is hard to evaluate since no measurements are recorded before the diagnosis. Secondary measurements concerning nutritional status such
Fig. 1 Kaplan-Meier survival curve stratified by esophagectomy with or without nutritional jejunostomy for patients treated for esophageal or gastro-esophageal junction cancer $(P=0.417)$

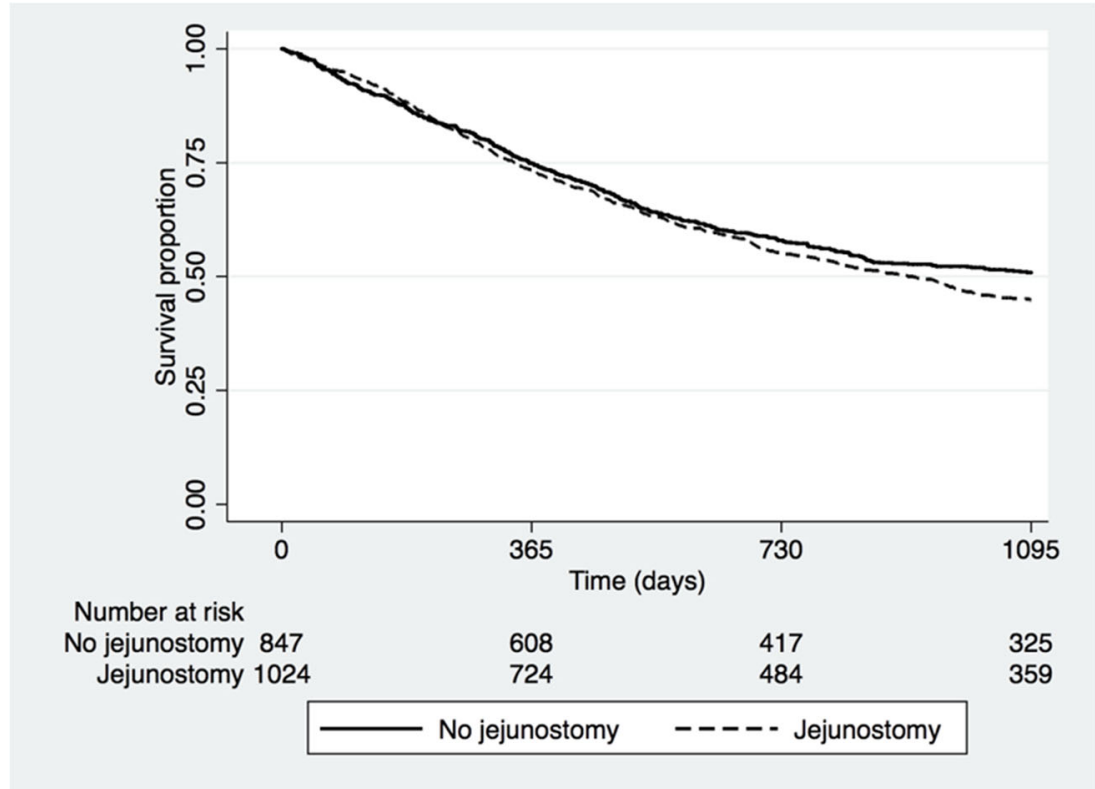


Table 5 Risk for Clavien-Dindo score $\geq$ IIIb and 90-day mortality comparing patients with jejunostomy vs. no jejunostomy in patients with postoperative complications after esophagectomy for cancer

\begin{tabular}{|c|c|c|c|}
\hline & \multicolumn{3}{|c|}{ Odds ratio ( $95 \%$ confidence interval) ${ }^{\dagger}$} \\
\hline \multicolumn{4}{|l|}{ Clavien-Dindo score $\geq \mathrm{IIIb}$} \\
\hline Any complication & 1.0 (reference) & $1.09(0.72-1.65)$ & 0.689 \\
\hline Surgical complication & 1.0 (reference) & $1.07(0.62-1.85)$ & 0.815 \\
\hline Anastomotic leak & 1.0 (reference) & $0.19(0.04-0.94)$ & 0.041 \\
\hline Chylothorax & 1.0 (reference) & $0.67(0.10-4.51)$ & 0.682 \\
\hline Recurrent laryngeal nerve paralysis & 1.0 (reference) & $1.48(0.29-7.51)$ & 0.633 \\
\hline Non-surgical complication & 1.0 (reference) & $1.56(0.92-2.63)$ & 0.099 \\
\hline Cardiovascular complication & 1.0 (reference) & $2.24(0.66-7.57)$ & 0.196 \\
\hline Pulmonary embolism & 1.0 (reference) & $0.80(0.15-4.12)$ & 0.788 \\
\hline Pneumonia & 1.0 (reference) & $1.64(0.71-3.79)$ & 0.248 \\
\hline Septicemia & 1.0 (reference) & $0.82(0.16-4.30)$ & 0.817 \\
\hline \multicolumn{4}{|l|}{ 90-day mortality } \\
\hline Any complication & 1.0 (reference) & $0.85(0.53-1.36)$ & 0.498 \\
\hline Surgical complication & 1.0 (reference) & $0.71(0.40-1.25)$ & 0.238 \\
\hline Anastomotic leak & 1.0 (reference) & $0.53(0.24-1.19)$ & 0.125 \\
\hline Chylothorax & 1.0 (reference) & $0.21(0.03-1.33)$ & 0.098 \\
\hline Recurrent nerve paralysis & 1.0 (reference) & $0.59(0.03-13.49)$ & 0.740 \\
\hline Non-surgical complication & 1.0 (reference) & $0.76(0.44-1.29)$ & 0.309 \\
\hline Cardiovascular complication & 1.0 (reference) & $0.95(0.31-2.88)$ & 0.922 \\
\hline Pulmonary embolism & 1.0 (reference) & $0.86(0.13-5.87)$ & 0.877 \\
\hline Pneumonia & 1.0 (reference) & $0.68(0.26-1.78)$ & 0.431 \\
\hline Septicemia & 1.0 (reference) & $0.74(0.26-2.09)$ & 0.572 \\
\hline
\end{tabular}

$\dagger$ Adjusted for histological tumor type, clinical tumor stage, and American Society of Anesthesiologists Score as mean body weight and baseline dysphagia score are however registered and were similar between the two groups. From the accessible data, preoperative nutritional status seems to have a minor impact on the decision to provide patients with a jejunostomy; local protocols are likely to have a more important role. It is however worth considering the risk for selection bias in the study.

Perioperative management including postoperative nutritional details or information about nasogastric tubes or early feeding is unfortunately not included in the register data. This study has analyzed the effects of the insertion of a jejunostomy at the time of the esophagectomy. A future study with more detailed data about oral, enteral, and parenteral nutrition after surgery is planned within our group.

Jejunostomy treatment details such as duration of catheter placement, degree of jejunostomy utilization, and minor jejunostomy-related complications would have been valuable to analyze, but this level of granularity of data is unfortunately not recorded in the register. However, no difference in surgical complications, Clavien-Dindo score, or re-operation was observed between the groups, which indicates that jejunostomy was not associated with increased risk for significant postoperative surgical complications.

Clavien-Dindo score was included in the register from 2012, and consequently, $211 / 784$ (26.9\%) of the patients with complication had missing data concerning Clavien-Dindo score. This is a weakness of the study; however, it is likely that this is proportionally distributed randomly between the groups. Missing data concerning tumor stage, surgical technique, and histological tumor type was taken into consideration as a complete case analysis was performed in the multivariable-adjusted model.

Strengths of the study include the population-based design, a relatively large cohort with a near-complete national coverage of all patients who underwent surgical resection for esophageal cancer in Sweden during the study period, small numbers of missing data, and the complete follow-up concerning survival made possible by the use of the National Cause of Death Register [19].

Previous research has shown that nutritional jejunostomies as part of curative treatment of esophageal cancer are safe, but controversy exists on the practice of routinely doing so, as evidence of its benefits in general is lacking [20, 23]. The nutritional deficit, weight loss, and sarcopenia are major issues that require intervention for patients undergoing esophageal cancer treatment. Jejunostomy insertion before the start of neoadjuvant treatment might provide an even more efficient nutritional treatment in selected patients [21, 24]. It is, however, challenging to design high-quality studies about nutritional treatments. Future studies need to focus on identifying 
patients who may benefit most from a nutritional jejunostomy, timing of placement, how and when it should be used, and also monitor changes in body composition prior to and during multimodality treatment, preferably in a randomized design.

In conclusion, this study indicates that a nutritional jejunostomy might decrease the risk for severe outcomes in patients with postoperative anastomotic leak after esophagectomy. However, our data suggests no clear benefit to apply standardized nutritional jejunostomy to all esophagectomy patients. Future research needs to investigate the optimal use of nutritional jejunostomy in esophageal cancer treatment.

Authors' contributions Study conception and design: Holmén, Hayami, Szabo, Rouvelas, Agustsson, Klevebro; acquisition of data: Holmén, Hayami, Rouvelas, Klevebro; analysis and interpretation of data: Holmén, Hayami, Agustsson, Klevebro; drafting of manuscript: Holmén, Klevebro, Agustsson, Rouvelas; critical revision of manuscript: Holmén, Hayami, Szabo, Rouvelas, Agustsson, Klevebro.

Funding Open access funding provided by Karolinska Institutet. This work was supported by grants provided by Region Stockholm.

\section{Compliance with ethical standards}

Conflict of interest The authors have declared no conflicts of interest.

Ethical approval All procedures performed in studies involving human participants were in accordance with the ethical standards of the institutional and/or national research committee and with the 1964 Helsinki declaration and its later amendments or comparable ethical standards. Approval for the study was granted by the Regional Ethical Review Board in Stockholm, Sweden (Dnr 2013/596-31/3).

Informed consent For the present study, no informed consent was required. This was approved by the Regional Ethical Review Board in Stockholm, Sweden.

Open Access This article is licensed under a Creative Commons Attribution 4.0 International License, which permits use, sharing, adaptation, distribution and reproduction in any medium or format, as long as you give appropriate credit to the original author(s) and the source, provide a link to the Creative Commons licence, and indicate if changes were made. The images or other third party material in this article are included in the article's Creative Commons licence, unless indicated otherwise in a credit line to the material. If material is not included in the article's Creative Commons licence and your intended use is not permitted by statutory regulation or exceeds the permitted use, you will need to obtain permission directly from the copyright holder. To view a copy of this licence, visit http://creativecommons.org/licenses/by/4.0/.

\section{References}

1. Low DE, Kuppusamy MK, Alderson D, Cecconello I, Chang AC, Darling G, Davies A, D'Journo XB, Gisbertz SS, Griffin SM, Hardwick R, Hoelscher A, Hofstetter W, Jobe B, Kitagawa Y, Law S, Mariette C, Maynard N, Morse CR, Nafteux P, Pera M, Pramesh CS, Puig S, Reynolds JV, Schroeder W, Smithers M, Wijnhoven BPL (2019) Benchmarking complications associated with esophagectomy. Ann Surg 269:291-298. https://doi.org/10. 1097/SLA.0000000000002611

2. Evans RPT, Singh P, Nepogodiev D, Bundred J, Kamarajah S, Jefferies B, Siaw-Acheampong K, Wanigasooriya K, McKay S, Mohamed I, Whitehouse T, Alderson D, Gossage J, van Hillegersberg R, Vohra RS, Griffiths EA (2019) Study protocol for a multicenter prospective cohort study on esophagogastric anastomoses and anastomotic leak (the Oesophago-Gastric Anastomosis Audit/OGAA). Dis Esophagus Off J Int Soc Dis Esophagus. https://doi.org/10.1093/dote/doz007

3. Markar S, Gronnier C, Duhamel A, Mabrut JY, Bail JP, Carrere N, Lefevre JH, Brigand C, Vaillant JC, Adham M, Msika S, Demartines N, Nakadi IE, Meunier B, Collet D, Mariette C, FREGAT (French Eso-Gastric Tumors) working group, FRENCH (Fédération de Recherche EN CHirurgie), and AFC (Association Française de Chirurgie) (2015) The impact of severe anastomotic leak on long-term survival and cancer recurrence after surgical resection for esophageal malignancy. Ann Surg 262:972980. https://doi.org/10.1097/SLA.0000000000001011

4. Schieman C, Wigle DA, Deschamps C, Nichols III FC, Cassivi SD, Shen KR, Allen MS (2012) Patterns of operative mortality following esophagectomy. Dis Esophagus Off J Int Soc Dis Esophagus 25:645-651. https://doi.org/10.1111/j.1442-2050.2011.01304.x

5. Derogar M, Orsini N, Sadr-Azodi O, Lagergren P (2012) Influence of major postoperative complications on health-related quality of life among long-term survivors of esophageal cancer surgery. J Clin Oncol Off J Am Soc Clin Oncol 30:1615-1619. https://doi.org/10. 1200/JCO.2011.40.3568

6. Kauppila J, Johar A, Lagergren P (2018) Postoperative complications and health-related quality of life 10 years after esophageal cancer surgery. Ann Surg Publish Ahead of Print 271:311-316. https://doi.org/10.1097/SLA.0000000000002972

7. Markar SR, Schmidt H, Kunz S, Bodnar A, Hubka M, Low DE (2014) Evolution of standardized clinical pathways: refining multidisciplinary care and process to improve outcomes of the surgical treatment of esophageal cancer. J Gastrointest Surg Off J Soc Surg Aliment Tract 18:1238-1246. https://doi.org/10.1007/s11605-0142520-6

8. Blencowe NS, Strong S, McNair AGK et al (2012) Reporting of short-term clinical outcomes after esophagectomy: a systematic review. Ann Surg 255:658-666. https://doi.org/10.1097/SLA. 0b013e3182480a6a

9. Kingma BF, Steenhagen E, Ruurda JP, van Hillegersberg R (2017) Nutritional aspects of enhanced recovery after esophagectomy with gastric conduit reconstruction. J Surg Oncol 116:623-629. https:// doi.org/10.1002/jso.24827

10. Koyanagi K, Tachimori Y (2017) Early oral nutrition plays an active role in enhanced recovery after minimally invasive esophagectomy. J Thorac Dis 9:3598-3602. https://doi.org/10.21037/jtd. 2017.09.26

11. Braga M, Gianotti L, Gentilini O, Parisi V, Salis C, di Carlo V (2001) Early postoperative enteral nutrition improves gut oxygenation and reduces costs compared with total parenteral nutrition. Crit Care Med 29:242-248. https://doi.org/10.1097/00003246200102000-00003

12. Han H, Pan M, Tao Y, Liu R, Huang Z, Piccolo K, Zhong C, Liu R (2018) Early enteral nutrition is associated with faster postesophagectomy recovery in Chinese esophageal cancer patients: a retrospective cohort study. Nutr Cancer 70:221-228. https://doi. org/10.1080/01635581.2018.1412477

13. Lorimer PD, Motz BM, Watson M, Trufan SJ, Prabhu RS, Hill JS, Salo JC (2019) Enteral feeding access has an impact on outcomes for patients with esophageal cancer undergoing esophagectomy: an analysis of SEER-Medicare. Ann Surg Oncol 26:1311-1319. https://doi.org/10.1245/s10434-019-07230-0 
14. Berkelmans GH, van Workum F, Weijs TJ et al (2017) The feeding route after esophagectomy: a review of literature. J Thorac Dis 9: S785-S791. https://doi.org/10.21037/jtd.2017.03.152

15. Sethuraman SA, Dhar VK, Habib DA, Sussman JE, Ahmad SA, Shah SA, Tsuei BJ, Sussman JJ, Abbott DE (2017) Tube feed necrosis after major gastrointestinal oncologic surgery: institutional lessons and a review of the literature. J Gastrointest Surg Off J Soc Surg Aliment Tract 21:2075-2082. https://doi.org/10.1007/s11605017-3593-9

16. Afaneh C, Gerszberg D, Slattery E, Seres DS, Chabot JA, Kluger MD (2015) Pancreatic cancer surgery and nutrition management: a review of the current literature. Hepatobiliary Surg Nutr 4:59-71. https://doi.org/10.3978/j.issn.2304-3881.2014.08.07

17. Dholaria S, Lakhera KK, Patni S (2017) Intussusception: a rare complication after feeding jejunostomy; a case report. Indian $\mathrm{J}$ Surg Oncol 8:188-190. https://doi.org/10.1007/s13193-016-06043

18. Linder G, Lindblad M, Djerf P, Elbe P, Johansson J, Lundell L, Hedberg J (2016) Validation of data quality in the Swedish National Register for Oesophageal and Gastric Cancer. Br J Surg 103:13261335. https://doi.org/10.1002/bjs.10234

19. Ludvigsson JF, Almqvist C, Edstedt Bonamy A-K et al (2017) Registers of the Swedish total population and their use in medical research. Eur J Epidemiol 31:125-136

20. Klevebro F, Johar A, Lagergren J, Lagergren P (2018) Outcomes of nutritional jejunostomy in the curative treatment of esophageal cancer. Dis Esophagus Off J Int Soc Dis Esophagus. https://doi. org/10.1093/dote/doy113

21. Dalton BGA, Friedant AJ, Su S, Schatz TAP, Ruth KJ, Scott WJ (2017) Benefits of supplemental jejunostomy tube feeding during neoadjuvant therapy in patients with locally advanced, potentially resectable esophageal cancer. J Laparoendosc Adv Surg Tech A 27: 1279-1283. https://doi.org/10.1089/lap.2017.0320

22. Koterazawa Y, Oshikiri T, Hasegawa H, Yamamoto M, Kanaji S, Yamashita K, Matsuda T, Nakamura T, Suzuki S, Kakeji Y (2019) Routine placement of feeding jejunostomy tube during esophagectomy increases postoperative complications and does not improve postoperative malnutrition. Dis Esophagus Off J Int Soc Dis Esophagus. https://doi.org/10.1093/dote/doz021

23. Weijs TJ, van Eden HWJ, Ruurda JP et al (2017) Routine jejunostomy tube feeding following esophagectomy. J Thorac Dis 9:S851-S860. https://doi.org/10.21037/jtd.2017.06.73

24. Jenkins TK, Lopez AN, Sarosi GA, Ben-David K, Thomas RM (2018) Preoperative enteral access is not necessary prior to multimodality treatment of esophageal cancer. Surgery 163:770776. https://doi.org/10.1016/j.surg.2017.09.046

Publisher's note Springer Nature remains neutral with regard to jurisdictional claims in published maps and institutional affiliations. 